\section{Desigualdades sociais e homicídios na cidade de São \\ Paulo, 1998}

\section{Homicide and social inequalities in the city of São Paulo, 1998}

Rita Barradas Barata'

Manoel Carlos Sampaio de Almeida Ribeiro'

Meri De Sordi²

${ }^{1}$ Departamento de Medicina Social da Faculdade de Ciências Médicas da Santa Casa de São Paulo

${ }^{2}$ Centro de Estudos Augusto Leopoldo Ayrosa Galvão (CEALAG)

Financiamento: Projeto financiado pela FAPESP, Processo99/06273-0

Agradecimentos: os autores agradecem o trabalho dedicado de Maria Carolina de Toledo Sivieri, Pedro Areno, Stella Velasques Anderaos Cassi na localização dos boletins de ocorrência e de Otávio Cabrera de Léo, Thiago Carvalho Pelucio Silva e Mariana Galvão Nascimento na análise dos inquéritos policiais.

Correspondência: Rita Barradas Barata, Rua Dr. Cesário Motta Jr. $616^{\circ}$ andar. Cep 01221-020. Email: rita.barata@fcmscsp.edu.br

\section{Resumo}

Este estudo teve como objetivo identificar os padrões de ocorrência dos homicídios quanto a características dos agressores, das vítimas e das circunstâncias que motivaram a agressão, segundo estratos de condições de vida. As condições de vida foram avaliadas pela escolaridade, renda e condições de habitação. Os distritos administrativos da cidade foram agrupados através de análise de cluster formando cinco estratos. Amostras casuais simples de 100 óbitos por homicídios foram obtidas para cada estrato. Atestado de óbito, boletim de ocorrência e inquérito policial foram utilizados como fontes de dados. No estrato A foram mais comuns os homicídios associados a assaltos, enquanto nos estratos B, C e D predominaram os homicídios relacionados ao tráfico de drogas e a outras atividades ilegais. No estrato E, a maioria dos casos estudados estava relacionada com brigas e desavenças entre indivíduos. Vítimas e os agressores foram predominantemente indivíduos do sexo masculino, jovens, brancos ou pardos, com baixa escolaridade, nascidos em São Paulo ou em Estados do Nordeste, com ocupação no setor terciário ou desempregados. Há maior freqüência relativa de homicídios de mulheres no estrato A. Neste estrato, a maioria das vítimas não conhece os agressores, enquanto nos outros estratos, predomina o conhecimento entre vítima e agressor envolvendo parentes, amigos ou conhecidos.

Palavras chave: Homicídio. Violência. Desigualdades sociais. Condições sociais.

O projeto foi aprovado pelo Comitê de Ética em Pesquisa da Santa Casa de São Paulo, № 062/00. 
Abstract

This study aimed to identify patterns in the occurrence of homicides considering the characteristics of aggressors, victims and the circumstances that motivated the aggression, according to life condition strata. Life conditions were evaluated according to schooling, income and housing conditions. We used a simple random sample of the deaths by homicides in each stratum of life conditions assessed by cluster analysis. Date sources: death certificates, police reports and police investigations. In stratum A, homicides were more commonly associated with robbery; while in strata $B$, $\mathrm{C}$ and $\mathrm{D}$, homicides were related to traffic and other illegal activities. In stratum E, most of the cases studied were related to fights and disagreements between individuals. Both victims and aggressors were male, young, white or "mixed race", with little schooling, born in São Paulo or in northeastern states, with an occupation in the third sector or unemployed. There was a greater relative frequency of homicides of women in strata A. In this stratum, the majority of victims did not know the aggressors, while in other strata, the link between victim and aggressor involving known relatives, friends or acquaintances prevailed.

Keywords: Homicide. Violence. Inequalities in health. Social conditions.

\section{Introdução}

As mortes e traumatismos decorrentes da violência vêm aumentando de maneira alarmante, a partir da década de 80 , convertendo-se em problema de saúde pública, seja por sua magnitude e transcendência social, seja pelo volume de gastos que $o$ atendimento das vítimas representa para o setor saúde ${ }^{1,2}$. A mortalidade por causas violentas tem sido responsável pelo aumento da mortalidade geral em muitos países latino-americanos e responde pela maior proporção de anos potenciais de vida perdidos ${ }^{3}$.

A mortalidade por homicídios representa uma das facetas do crescimento da violência urbana ocupando lugar de destaque entre as causas básicas de óbito, principalmente entre adolescentes e adultos jovens do sexo masculino, em muitos países da América, constituindo, ao lado dos acidentes de trânsito, os principais motivos das mortes por causas externas ${ }^{4-6}$.

Em estudo anterior, os autores analisaram a influência da concentração de renda nas taxas de mortalidade por homicídio nos municípios do Estado de São Paulo, encontrando taxas mais altas nos municípios com maiores populações urbanas, mais ricos (renda familiar média) e mais desiguais (relação entre a renda do percentil 90 e do percentil 20 da população e o índice de Gini) ${ }^{7}$.

Para aprofundar a análise do processo de determinação social da mortalidade por homicídios na cidade de São Paulo, este estudo teve como objetivo principal identificar os padrões de ocorrência dos homicídios quanto às características dos agressores, das vítimas e das circunstâncias que motivaram a agressão, segundo estratos populacionais que apresentam condições de vida bastante diferenciadas.

\section{Métodos}

O desenho adotado na pesquisa pode ser caracterizado como ecológico, no qual a variável de exposição - condições de 
vida - é medida em nível de agregado (estratos) e o desfecho - homicídio com as características da vítima, do agressor e circunstâncias - é medido nos indivíduos. Este tipo de abordagem visa identificar efeitos do contexto sobre os fenômenos analisados.

Para a estratificação dos distritos da cidade de São Paulo, segundo condições de vida, optou-se pela técnica de análise de "clusters" ou conglomerados, técnica apropriada para agrupar elementos de tal forma que cada conglomerado seja, por um lado, o mais homogêneo possível internamente, e, por outro, o mais diferente possível em relação aos demais conglomerados. A análise de conglomerados ou “clusters" baseia-se no cálculo de vetores resultantes da disposição de $\mathrm{n}$ elementos (neste caso os 96 distritos) no espaço de $\mathrm{p}$ dimensões ( $p$ é o número de variáveis selecionadas para a análise). A distância euclidiana foi escolhida como medida de proximidade e a técnica de k-médias para a conformação dos clusters (aplicativo SPSS 8.0).

Para caracterizar as condições de vida foram selecionados três componentes principais, para os quais existiam informações disponíveis no censo demográfico de 2000: escolaridade (taxa de analfabetismo em maiores de 5 anos, percentagem de chefes de família com menos de 4 anos de escolaridade e percentagem de chefes de família com mais de 10 anos de escolaridade), renda (renda média mensal, percentagem de pessoas com renda média mensal inferior a $1 / 2$ salário mínimo e percentagem de pessoas com renda média mensal superior a 20 salários mínimos) e condições de habitação (aglomeração intradomiciliar, percentagem de domicílios servidos por rede de água e rede de esgoto). Todos os valores das variáveis foram transformados em valores de $\mathrm{z}$ para evitar a influência das escalas de medida.

\section{Amostra}

O universo amostral foi constituído por todos os óbitos de pessoas residentes no município de São Paulo, ocorridos em 1998, cuja causa básica tenha sido homicídio (códigos entre X850 e Y090 da CID 10), obtidos junto à Fundação SEADE. Estes óbitos foram classificados segundo os estratos de condições de vida a partir da residência da vítima.

Para um universo estimado de 5.000 óbitos por homicídios esperava-se que $16 \%$ dos mesmos resultassem em inquéritos policiais inconclusivos, restando 4.200 para os quais as informações desejadas seriam disponíveis ${ }^{4}$. Assumindo um efeito do desenho igual a dois, erro alfa de $1 \%$, erro beta de $90 \%$ e prevalência de $50 \%$ em média para as diferentes características de interesse, seriam necessários 50 óbitos por estrato. Considerando as dificuldades de obtenção das informações e as possíveis perdas, optou-se por dobrar o número de casos a serem incluídos, chegando a 500 . Tendo em vista a distribuição desigual entre os estratos e o objetivo de comparar características entre os estratos optou-se por incluir 100 óbitos para cada estrato. Devido a perdas na localização dos atestados de óbito, a amostra final ficou com 486 homicídios ao invés dos 500 inicialmente sorteados. Não foi feita a reposição para não alterar o procedimento de aleatorização.

\section{Fontes de informação}

Em 241 casos, o único documento localizado foi a declaração de óbito. A Figura 1 mostra a distribuição percentual de atestados de óbito, boletins de ocorrência e inquéritos policiais por estrato.

Os números correspondentes aos boletins de ocorrência foram levantados no Instituto Médico Legal, e em seguida os documentos foram buscados manualmente nas seccionais de polícia. Foram localizados apenas 195 (40\%) boletins correspondentes aos 486 atestados de óbitos incluídos na amostra. A maioria desses documentos, entretanto, traz poucas informações sobre os agressores e as circuns- 
Figura 1 - Distribuição percentual dos atestados de óbito, boletins de ocorrência e inquéritos policiais para os homicídios estudados segundo estrato de condições de vida, São Paulo, 1998. Figure 1 - Distribution of percentages of death certificates, police reports and police investigations for homicides studied according to life condition stratum, São Paulo, 1998.

\begin{tabular}{lccc}
\hline Estrato & Atestado de óbito & Boletim de ocorrência & Inquérito policial \\
\hline A & 94 & 44 & 27 \\
B & 93 & 31 & 29 \\
C & 95 & 33 & 37 \\
D & 100 & 34 & 15 \\
E & 100 & 50 & 13 \\
\hline
\end{tabular}

Fonte: Fundação SEADE

Source: SEADE Foundation

tâncias dos homicídios, pois grande parte das informações de interesse não é preenchida.

Os inquéritos policiais foram buscados junto à Delegacia de Homicídios e Proteção às Pessoas (DHPP). No entanto, a busca não surtiu resultados, levando a equipe a solicitar autorização aos juízes responsáveis pelos tribunais de júri em São Paulo para a localização dos inquéritos. Este processo de localização foi extremamente moroso, visto que o sistema não está informatizado e as declarações de óbito dispõem apenas do nome da vítima e não do agressor ou réu. Deste modo, foram estudados apenas 115 inquéritos (46,9\%) correspondentes aos homicídios que compõem a amostra. O preenchimento dos dados de interesse foi também bastante demorado, pois os inquéritos demandaram bastante tempo para ser lidos e corretamente compreendidos. O prazo de conclusão do projeto não permitiu seguir buscando os outros inquéritos e a análise apresentada baseou-se apenas nestes.

Tendo em vista as limitações impostas pelas fontes de dados e pela estratégia de amostragem adotada, não é possível considerar que os dados analisados sejam representativos da população de São Paulo. Portanto, a apresentação e discussão dos resultados terão caráter exploratório, baseando-se no padrão qualitativo identificado, não sendo possível realizar inferências estatísticas válidas.

\section{Resultados}

A distribuição das taxas de mortalidade por homicídios em 1998, segundo os estratos de condições de vida, podem ser observadas na Figura 2. As taxas aumentam conforme pioram as condições de vida nos distritos de residência das vítimas. Utilizando o estrato A como referência, observa-se risco crescente do melhor para o pior estrato.

\section{Características das vítimas}

A Figura 3 apresenta as principais características das vítimas de homicídio. Segundo os estratos de condições de vida, não houve diferença significativa para estado civil, condição migratória, ocupação e antecedentes criminais.

A proporção de óbitos foi sempre maior para o sexo masculino, variando entre $84 \%$ dos óbitos no estrato A e $97 \%$ no estrato E. A proporção de óbitos em mulheres foi significantemente maior no estrato A ( $p<0,007)$. A razão entre os homicídios masculinos e femininos atingiu valor máximo - 32 óbitos masculinos para cada óbito feminino - no estrato E. A menor relação foi observada no estrato A, no qual para cada homicídio feminino ocorreram 5,1 homicídios masculinos.

Com relação à idade também foram observadas diferenças estatisticamente significantes entre os estratos. A análise de variância entre as médias apontou diferen- 
Figura 3 - Características das vítimas de homicídio, São Paulo, 1998.

Figure 3 - Characteristics of homicide victims, São Paulo, 1998.

\begin{tabular}{lcc}
\hline Variáveis & Ocorrência & IC95\% \\
\hline Estado civil: solteiros & 75,0 & $71,5-79,3$ \\
Sexo masculino & 92,1 & $89,7-94,5$ \\
Idade: 20 - 29 anos & 63,7 & $59,4-68,0$ \\
Cor branca & 53,4 & $48,9-57,9$ \\
Nascidos em São Paulo & 54,7 & $50,2-59,2$ \\
Instrução primária & 71,2 & $67,1-75,3$ \\
Ocupação & & \\
- comercio/prestação serviços & 45,1 & $40,7-49,5$ \\
- atividades criminais & 11,5 & $8,7-14,3$ \\
- desempregados & 10,5 & $7,7-13,2$ \\
Antecedentes criminais & 34,2 & $25,0-43,0$ \\
Abuso de drogas & & \\
- álcool & 6,5 & $4,7-8,3$ \\
- crack, cocaína ou maconha & 27,8 & $21,5-34,1$ \\
- nenhuma & 27,8 & $21,5-34,1$ \\
\hline
\end{tabular}

ças significantes entre os estratos ( $\mathrm{p}<$ 0.004). Tanto para o estrato A quanto para o estrato B, as vítimas são em média mais velhas do que as vítimas residentes nas demais áreas.

Observa-se aumento proporcional crescente das vítimas negras e de cor parda à medida que se passa do estrato $A$ para o estrato E. A proporção de brancos entre as vítimas residentes no estrato A é 1,58 vezes maior do que a proporção de brancos entre as vítimas residentes no estrato E. Proporcionalmente houve mais vítimas com instrução secundária e superior entre os moradores do estrato A do que nos demais. Predomina amplamente, em todos os estratos, os indivíduos com instrução primária. O consumo de álcool e drogas ilícitas apresenta proporções crescentes à medida que se vai do estrato A para o estrato E, passando de cerca de $30 \%$ a $75 \%$ nesse último estrato.

\section{Características dos agressores}

Com relação ao número de agressores envolvidos no homicídio, em 59,3\% dos casos houve apenas um agressor e em
40,7\% múltiplos agressores, sendo mais freqüente a presença de dois agressores. $\mathrm{O}$ número máximo registrado foi de 5 agressores por episódio. O sexo dos agressores foi desconhecido em $30 \%$ dos casos. Nos demais o agressor foi do sexo masculino em $67,1 \%$ dos casos.

Para todas as variáveis analisadas havia falta de informações na maioria dos documentos pesquisados. Dentre aqueles com informações conhecidas (variando de 30 a $60 \%$ segundo a variável considerada), as características predominantes foram sexo masculino, idade entre 20 e 39 anos (média de 28 anos e mediana de 24 anos), 23\% com antecedentes criminais, sem informações sobre uso de álcool ou droga, policiais militares, comerciantes ou desempregados, de cor branca, com instrução primária.

Proporção extremamente alta dos homicídios tem autoria desconhecida, impedindo assim, que se trace um perfil adequado dos criminosos.

\section{Características dos homicídios}

A Figura 4 apresenta as principais características dos homicídios e das circuns- 
tâncias nas quais eles ocorreram. Em todos os estratos os homicídios se concentraram no período de 18:00 às 24:00 horas e das 0:00 às 6:00 horas.

A maioria dos óbitos ocorreu em hospitais, mas a proporção variou entre $65,0 \%$ para os do estrato A e $48,5 \%$ para o estrato E. Para os óbitos em via pública observouse um crescimento proporcional passando de $18 \%$ no estrato A para $40 \%$ no estrato E. O inverso foi observado para os óbitos no domicílio, com 9\% no estrato A caindo para $2 \%$ no estrato D. No conjunto da amostra a razão entre óbitos ocorridos nos hospitais foi de 19,2 óbitos masculinos para 1 feminino. Em via pública ocorreram 9,5 óbitos masculinos para cada óbito feminino e no domicílio da vítima foram 2,1 óbitos masculinos para cada óbito feminino.

No estrato A houve maior freqüência de homicídios cujos óbitos ocorreram no domicílio da vítima e $54 \%$ destes casos afetaram indivíduos do sexo feminino. Nos demais estratos, para todos os locais de ocorrência do óbito houve predomínio do sexo masculino. As demais características, ex-

Figura 4 - Características dos homicídios e das circunstâncias em que ocorreram, São Paulo, 1998

Figure 4 - Characteristics of homicides and circumstances in which they occurred, São Paulo, 1998

\begin{tabular}{|c|c|c|}
\hline Variáveis & Ocorrência & IC 95\% \\
\hline \multicolumn{3}{|l|}{ Local } \\
\hline - espaços públicos & 65,1 & $64,3-65,9$ \\
\hline - bares e outros estabelecimentos & 15,1 & $13,8-16,4$ \\
\hline - domicílio & 9,1 & $7,7-10,4$ \\
\hline \multicolumn{3}{|l|}{ Tipo } \\
\hline - único & 85,5 & $84,9-86,0$ \\
\hline - chacina & 14,5 & $10,5-19,0$ \\
\hline \multicolumn{3}{|l|}{ Instrumentos } \\
\hline - armas de fogo & 83,3 & $82,9-83,7$ \\
\hline - armas brancas & 8,0 & $7,1-8,9$ \\
\hline \multicolumn{3}{|l|}{ Lesões } \\
\hline - hemorragias traumáticas & 49,2 & $48,6-49,8$ \\
\hline - ferimentos na cabeça & 35,9 & $35,2-36,6$ \\
\hline \multicolumn{3}{|l|}{ Motivo } \\
\hline - desconhecido & 35,8 & $34,3-37,3$ \\
\hline - tráfico/consumo de drogas & 19,4 & $17,7-21,0$ \\
\hline - brigas e outros motivos fúteis & 17,9 & $16,2-19,6$ \\
\hline - assaltos seguidos de morte & 10,4 & $8,7-12,1$ \\
\hline - vingança & 8,2 & $6,5-10,0$ \\
\hline \multicolumn{3}{|l|}{ Relação com o agressor } \\
\hline - desconhecida & 40,8 & $39,4-42,2$ \\
\hline - conhecidos, amigos ou parentes & 37,0 & $35,6-38,5$ \\
\hline - sem relação & 21,7 & $20,1-23,3$ \\
\hline \multicolumn{3}{|l|}{ Proximidade com a residência } \\
\hline - mesmo distrito administrativo & 59,4 & $58,8-60,0$ \\
\hline - distritos vizinhos & 18,3 & $17,5-19,1$ \\
\hline - distritos próximos na mesma região & 16,5 & $15,7-17,3$ \\
\hline - distritos distantes em outras regiões & 8,7 & $7,9-9,6$ \\
\hline \multicolumn{3}{|l|}{ Conseqüências para o agressor } \\
\hline - desconhecida & 53,2 & $52,0-54,5$ \\
\hline - sem identificação dos suspeitos & 27,9 & $26,3-29,5$ \\
\hline - suspeitos foragidos & 9,4 & $7,7-11,1$ \\
\hline - julgados e condenados & 3,6 & $1,8-5,4$ \\
\hline - assassinados & 3,4 & $1,6-5,2$ \\
\hline
\end{tabular}




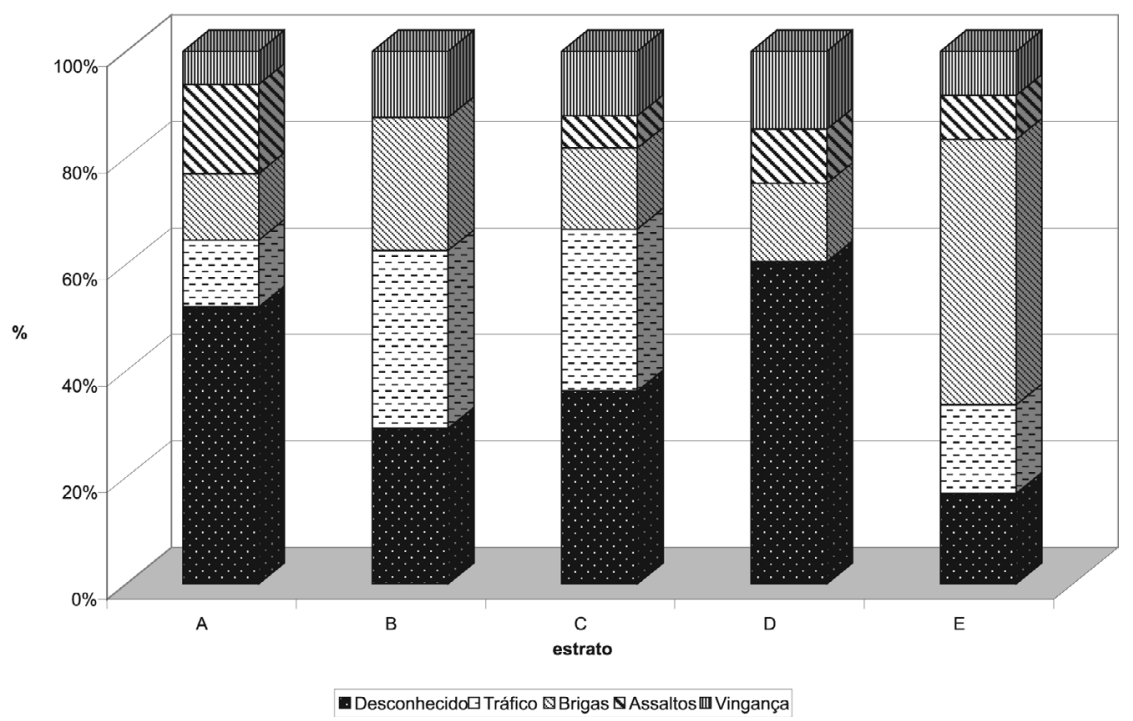

Figura 5- Distribuição dos motivos dos homicídios por estrato de condição de vida, SP,1998 Figure 5-Distribution of causes of homicides by life condition stratum

ceção feita aos motivos do homicídio, não mostraram diferenças significantes entre os estratos.

A Figura 5 apresenta a distribuição dos motivos para os homicídios segundo os estratos de condição de vida. Tomando o estrato E como referência, houve 3,2 vezes mais homicídios por razões desconhecidas no estrato $A, 1,4$ vezes no estrato $B, 2$ vezes no estrato $\mathrm{C}$ e 2,8 vezes no estrato $\mathrm{D}$. Os assaltos seguidos de homicídios foram 2 vezes mais freqüentes no estrato $\mathrm{A}$, menos freqüentes nos estratos B, C, e semelhantes no estrato D. Os homicídios relacionados com o tráfico de drogas foram 1,7 vezes mais freqüentes nos estratos $\mathrm{B}$ e $C$, não foram registrados no estrato $D$ e foram menos freqüentes no estrato A. Já os homicídios relacionados à vingança foram 1,2 a 1,4 vezes mais freqüentes nos estratos $B$, $\mathrm{C}$ e $\mathrm{D}$ e menos freqüentes no estrato $\mathrm{A}$.

\section{Discussão}

O primeiro aspecto importante a ser destacado é a enorme dificuldade que houve na obtenção das informações de interesse. A declaração de óbito foi o documento de mais fácil acesso e com informações mais completas, porém apresenta a limitação de trazer informações apenas sobre a vítima.

Os boletins de ocorrência mostraramse como a pior fonte de informações dentre as utilizadas. No ano de 1998, não havia ainda um sistema informatizado nem a determinação da Secretaria de Segurança para os legistas anotarem o número da declaração de óbito no boletim de ocorrência. Assim, a busca por esse documento demandou duas operações demoradas e pouco produtivas: a localização nos livros do Instituto Médico Legal do número do boletim de ocorrência correspondente a cada declaração sorteada e,depois, a localização dos referidos boletins nas seccionais da polícia. Todo o trabalho resultou em pouquíssimas informações úteis, dado que na maioria das vezes as informações buscadas não estavam anotadas. Após a criação do sistema de informações INFOCRIM, 92\% dos boletins de ocorrência são localizáveis ${ }^{8}$.

Os inquéritos policiais são uma fonte melhor de informações sobre as circunstâncias do homicídio e algumas das características dos agressores. Entretanto, a localização e o acesso aos inquéritos não se mostraram tarefa fácil, estando na dependência dos funcionários do poder judiciário. 
Assim, a pesquisa deparou-se com enormes problemas de qualidade e acesso às informações, colocando em questão o desenho que havia sido imaginado pelos pesquisadores. Para melhor responder às questões da pesquisa, provavelmente, melhor teria sido partir da identificação dos inquéritos referentes a homicídios de um determinado ano e então proceder à classificação dos mesmos por regiões e áreas da cidade, verificando a que proporção dos homicídios registrados, através dos atestados de óbito, esses casos correspondiam.

Drumond Jr. ${ }^{9}$, estudando a totalidade dos óbitos por homicídios ocorridos em São Paulo, em 1998, encontrou as mesmas características entre as vítimas apontadas neste estudo: homens, adultos jovens, com baixo nível de instrução, ocupação no setor industrial (construção civil) ou de serviços. A distribuição por idade, sexo e nível de instrução também se repetem para os óbitos de moradores da região metropolitana em $2000^{10}$. Estas características se assemelham àquelas observadas em grandes centros urbanos em outros estados do país e em diferentes países do continente durante a década de $90^{11-13}$.

Estudo conduzido em São Paulo, para óbitos de 2001, baseado em dados dos boletins de ocorrência e laudos de necropsia, apontam as mesmas proporções de uso de álcool e drogas pelas vítimas, encontradas neste estudo ${ }^{8}$.

Segundo Souza ${ }^{14}$, para os adolescentes e adultos jovens vivendo em condição de exclusão social, os comportamentos violentos aparecem como reafirmadores de identidade, levando este grupo etário a predominar entre os agentes e os alvos da violência. A frustração das expectativas, associada ao desemprego crônico e à falta de participação na vida social, acabam por produzir sentimentos de marginalização e diminuição da auto-estima, concorrendo para maior exposição e vitimização.

Estudo de caso-controle realizado em Recife, na década de 90, identificou como fatores associados ao risco de homicídio em crianças e adolescentes a presença de antecedentes criminais, o uso de drogas, baixa escolaridade, ausência da figura paterna e ausência de práticas religiosas, coincidindo com o perfil encontrado nesta pesquisa $^{15}$.

A utilização de armas de fogo como principal veículo para a perpetração dos homicídios é relatada pela maioria das pesquisas sobre o tema, anteriormente mencionadas. Em São Paulo, os vários estudos sobre homicídios mostram maior ocorrência nos espaços públicos, tais como bares e rua (cerca de $80 \%$ ), e pequena proporção de homicídios praticados nos domicílios das vítimas (menos de $10 \%)$. Em Nova York, cerca de 30\% dos homicídios ocorrem nos domicílios ou locais de trabalho, e $45 \%$ em locais públicos ${ }^{11}$.

Dadas as precariedades das fontes de dados, poucos são os estudos sobre as características dos agressores. O estudo realizado em Cali também encontrou informações para apenas 20 a $27 \%$ dos casos $^{13}$. Entretanto, os poucos dados disponíveis mostram que o perfil dos agressores não difere significantemente do perfil das vítimas. Este achado sugere que tornar-se vítima ou agressor, no caso das mortes por homicídio, depende mais de circunstâncias casuais do que de características dos indivíduos.

Quanto às circunstâncias que levaram ao homicídio, também as informações são escassas. Pesquisa realizada na Bahia identificou percentual semelhante aos desta pesquisa para homicídios relacionados com o tráfico de drogas, brigas e assaltos. Entretanto, houve proporção maior de homicídios por vingança e motivos passionais ${ }^{16}$.

Outro estudo baseado na análise de inquéritos policiais, realizado por Eva Blay $^{17}$ para analisar a violência contra as mulheres em São Paulo, com dados de 1998, encontrou proporção semelhante de agressores não identificados e de agressores que eram parentes, amigos ou conhecidos àquela observada no presente estudo. Um número significantemente 
maior de réus foi julgado e condenado nessa amostra composta apenas por casos em que as vítimas eram mulheres. A proporção de réus foragidos, impronunciados e absolvidos, no entanto, foi bastante parecida, demonstrando a impunidade que acompanha o crescimento da violência urbana em São Paulo.

A menor razão entre homicídios masculinos e femininos, no estrato de melhor nível socioeconômico, juntamente com a maior concentração de ocorrências nos domicílios, parece estar associada ao fato dos homicídios relacionados com assaltos se concentrarem nesta camada da população.

Observaram-se também diferenças significativas na distribuição etária das vítimas. A mesma foi inversamente proporcional às condições socioeconômicas. A maior proporção de vítimas de cor negra nos estratos de piores condições socioeconômicas reflete a composição populacional. O mapa da juventude mostra que as áreas mais periféricas da cidade, que são também as mais pobres e com piores condições de vida, apresentam maior concentração de população negra ${ }^{18}$.

Diferentes perspectivas teóricas sugerem que fatores de vizinhança ou de contexto poderiam estar associados com a mortalidade por homicídio apontando como possíveis explicações: a) a concentração da pobreza e as desigualdades econômicas impedindo ou frustrando a realização de aspirações, o acesso a bons empregos e a ganhos financeiros, e gerando condições sociais com impacto negativo nas condições de vida dos moradores; b) a concentração de famílias fraturadas e a segregação, racial, étnica ou de classe social associadas a pequeno grau de controle social, menor presença da polícia, prédios danificados, alto consumo de álcool e outras drogas. Por outro lado, bairros com altas concentrações de migrantes onde predominam fortes laços de solidariedade entre as famílias podem proteger contra a violência e os homicídios ${ }^{19}$.

Moser $^{20}$ identifica aspectos comuns aos vários países que apresentam taxas altas de mortalidade por homicídios nos quais há desconfiança na capacidade do Estado para controlar e prevenir o crime e a violência; expansão de alternativas não estatais de governança social, gerando formas nocivas de capital social e privatização da segurança pública.

Segundo Agudelo ${ }^{21}$, a violência como mecanismo para a solução de conflitos penetrou profundamente nos diversos estratos sociais, cenários e relações da sociedade colombiana. A convivência cotidiana com a violência transforma as relações sociais em relações baseadas na força, na coação e no medo. Esta cultura da violência se traduz em aumento da violência doméstica, na existência de "justiceiros" ou matadores de aluguel, expressando a negação total de qualquer ordenamento jurídico; massacres e assassinados coletivos e a percepção social de que existem populações socialmente descartáveis, como as prostitutas, os travestis, homossexuais, ladrões, mendigos, inválidos e os estrangeiros. Embora com menor freqüência, estes aspectos estão presentes também em outros países da região.

Apesar do crescimento da violência e de parcelas significativas da população dos grandes centros urbanos na América Latina viverem em áreas em que o cotidiano é violento, os indivíduos expostos a estas condições de vida podem responder de maneira distinta a ela. Klevens e Roca ${ }^{22}$ identificam três grupos de fatores protetores em situação de exposição a ambientes violentos: atributos pessoais como inteligência, auto-estima e sensibilidade; relações familiares calorosas, responsivas e responsáveis; e referências extra familiares ou suporte de adultos que forneçam modelos de identificação positivos.

A Comissão de Desenvolvimento Urbano e Interior da Câmara dos Deputados promoveu amplo debate sobre a violência no Brasil, em 2001, e as propostas para o enfrentamento da situação foram: desenvolver políticas públicas visando a inserção social dos jovens das camadas pobres 
da população através do aumento e melhoria de qualidade da escolaridade, criação de empregos e alternativas de lazer; reforçar as organizações de vizinhos potencializando o suporte social; controlar a comercialização e posse das armas de fogo; proteção às testemunhas de crimes violentos; policiamento ostensivo nas áreas mais violentas, sistemas de informações adequados para permitir articulação das ações policiais e maior conhecimento sobre o problema; discriminalizar o consumo de drogas; aumentar as penas para delitos graves.

\section{Conclusões}

Com relação às circunstâncias em que os homicídios foram cometidos, mesmo com poucos dados, as diferenças foram significantes entre os estratos. Evidentemente, não há segurança com relação à representatividade desses casos. Porém, qualitativamente, as histórias relacionadas com as tendências evidenciadas na análise quantitativa mostraram um padrão de repetição sugerindo sua representatividade. No estrato A foram mais comuns os homicídios associados a situações envolvendo assaltos, enquanto nos estratos B, C e D predominaram claramente os homicídios relacionados ao tráfico de drogas e, nos dois últimos estratos, os homicídios relacionados a outras atividades ilegais. Já no estrato E, a maioria dos casos estudados estava relacionada com brigas e desavenças entre indivíduos. Assim, pode-se concluir que as circunstâncias que cercam os homicídios variam entre os diferentes estratos de condição de vida.

Com relação às características sociodemográficas das vítimas e dos agressores, não somente há bastante semelhança entre elas num mesmo estrato, como entre os estratos. De maneira geral, as vítimas e os agressores são indivíduos do sexo masculino, jovens, brancos ou pardos, com baixa escolaridade, nascidos em São Paulo ou em Estados do Nordeste, com ocupação no setor terciário ou desempregados. Chama a atenção, apenas, a maior freqüência relativa de homicídios de mulheres nos estratos de melhores condições de vida.

Quanto às relações entre vítimas e agressores, como a grande maioria dos casos fica sem esclarecimento, não foi possível identificar padrões diferentes entre os estratos, para esse aspecto. Entretanto, tendo em vista as circunstâncias predominantes em cada estrato pode-se inferir que, no estrato $\mathrm{A}$, a maioria das vítimas não conhece os agressores, enquanto nos outros estratos, predominaria o conhecimento entre vítima e agressor, seja em função das atividades ligadas ao consumo e ao tráfico de drogas, seja nas situações de brigas e desavenças, geralmente envolvendo parentes, amigos ou conhecidos.

A impossibilidade de estabelecer padrões claramente distintos para a ocorrência dos homicídios em cada um dos estratos de condições de vida, além dos problemas relacionados com a obtenção dos dados para parcela maior dos homicídios estudados, relaciona-se possivelmente com a utilização do estrato como unidade de análise. Cada estrato reúne um conjunto amplo de distritos nos quais, em média, a população compartilha determinadas condições de vida. Entretanto, as vítimas de homicídio, em cada estrato podem não apresentar estas características médias.

Quanto à possível relação entre os homicídios e as desigualdades sociais existentes nessas áreas, dada a insuficiência de dados socioeconômicos referentes às vítimas e aos agressores nas fontes utilizadas, não foi possível investigar adequadamente essa questão. 


\section{Referências}

1. Jorge MHPM, Koizumi MS. Gastos governamentais do SUS com internações hospitalares por causas externas: análise no Estado de São Paulo 2000. Rev Bras Epidemiol 2004: 7(2): 228-38.

2. Gawryszewski VP, Jorge MHPM. Mortalidade violenta no município de São Paulo nos últimos 40 anos. Rev Bras Epidemiol 2000; 3(1-3): 50-69.

3. Butchart A, Engström K. Sex and age-specific relations between economic development, economic inequality and homicide rates in people aged 0-24 years: a crosssectional analysis. Bull World Health Organ 2002; 80(10): 797-805.

4. Gaweyszewski V, Mercy JA. Homicide trends and characteristics - Brazil,1980-2002. MMWR 2004; 53(8): 169-71.

5. Peres MFT. Violência por armas de fogo no Brasil. Núcleo de Estudos da Violência. USP; 2004

6. Briceño-León R. Urban violence and public health in Latin America: a sociological explanatory framework. Cad Saúde Pública 2005; 21(6): 1629-64.

7. Barata RB; Ribeiro MCSA. Relação entre homicídios e indicadores econômicos em São Paulo, Brasil,1996. Rev Panam Salud Pública 2000; 7(2): 118-24.

8. Gawryszewski VP, Kann T, Jorge MHPM. Informações sobre homicídios e sua integração com o setor saúde e segurança pública. Rev Saúde Pública 2005; 39(4): 627-33.

9. Drumond Jr. M. Homicídios e desigualdades sociais na cidade de São Paulo: uma visão epidemiológica. Saúde e Sociedade 1999; 8(1): 63-81.

10. Kilsztajn S, Carmo MSN, Sugahara GTL, Lopes ES. Vítimas da cor: homicídios na região metropolitana de São Paulo, Brasil, 2000. Cad Saúde Pública 2005; 21(5); 1408-15.

11. Tardiff K, Marzuk PM, Leon AC, Hirsch CS, Stajic M, Portera L, Hartwell N. A profile of homicides on the streets and in the homes of New York City. Public Health Rep 1995; 110(1): 13-7.
12. Briceño-León R. Buscando explicaciones a la violencia. Espacio abierto; 1997; 6(1): 45-69.

13. Concha-Eastman A, Espitia VE, Espinosa R, Guerrero R. La epidemiología de los homicidios en Cali 1993-1998: seis años de un modelo poblacional. Rev Panam Salud Pública 2002; 12(4): 230-9.

14. Souza ER. Masculinidade e violência no Brasil: contribuições para a reflexão no campo da saúde. Ciência e Saúde Coletiva 2005; 10(1): 59-70.

15. Falbo GH, Buzzetti R, Cattaneo A. Homicide in children and adolescents: a case-control study in Recife, Brazil. Bulletin of the WHO2001; 79(1): 2-7.

16. López MV, Híjar-Medina MC, Pacheco RAR, Muñoz JB. Muertes por homicídio, consecuencia fatal de la violencia. El caso de Mexico, 1979-1992. Rev Saúde Pública 1996; 30(1): 46-52.

17. Blay EA. Violência contra a mulher e políticas públicas. Estudos Avançados 2003; 17(49): 87-98.

18. Bousquat A, Cohn A. A construção do mapa da juventude. Lua Nova 2003; 60: 81-96.

19. Krueger PM, Huie SAB, Rogers RG, Hummer RA. Neighborhoods and homicide mortality: an analysis of race/ethnic differences. J Epidemiol Community Health 2004; 58: 223-30.

20. Moser CON. Urban violence and insecurity: an introductory roadmap. Environ Urbanization 2004; 16(2); 3-16.

21. Agudelo SF. Violencia y salud en Colombia. Rev Panam Salud Pública 1997; 1(2) :93-103.

22. Klevens J, Roca J. Nonviolent youth in a violent society: resilience and vulnerability in the country of Colombia. Violence and Victims 1999; 14(3): 311-22.

Recebido em: 23/01/07 Versão final reapresentada em: 30/08/07 Aprovado em: 04/12/07 